\title{
MOLECULAR WEIGHT DISTRIBUTION GENERATED ON INITIATION OF FREE RADICAL POLYMERIZATION BY SEQUENCE OF LASER PULSES
}

\author{
A. V. EVSEEV and A. N. NIKITIN \\ Research Centre for Technological Lasers, Russian Academy of \\ Sciences, Shatura, Moscow Region, 140700, Russia
}

(Received 5 September 1994)

\begin{abstract}
An investigation of molecular weight distributions (MWDs) resulting from initiation of free radical polymerization by an arbitrary sequence of short laser pulses has been undertaken. The analytical expressions have been derived to calculate MWDs for a polymerization scheme that contains reactions of chain initiation, propagation and termination by recombination or disproportionation. The MWDs produced by pulse-periodic irradiation have been calculated for a wide range of initiating pulses repetition rates $(f=0.05-100 \mathrm{~Hz})$. The MWD dynamics in the act of polymerization and the influence of the duration of polymerization pseudostationary regime establishment upon the MWD have also been studied. The suitability of the derived expressions for describing the MWD generated by CW radiation before and after the establishment of polymerization quasi-stationary regime has been considered.
\end{abstract}

KEY WORDS: Photopolymerization; pulsed laser polymerization; radical polymerization; molecular weight distribution; pseudostationary regime.

\section{INTRODUCTION}

Polymerization of vinyl monomers and oligomers photoinduced by pulsed laser radiation is an intensively studied process. ${ }^{1-11}$ The interest in this way of polymerization initiation is due both to the possibility of exerting easily control over energy input to the polymerization medium and, consequently, to some extent over the polymerization process in itself, and to a principal opportunity of extracting some information on this process kinetic parameters from the experiments on pulsed polymerization. The set of reaction constants that describe the polymerization process eventually governs molecular weight distribution (MWD) ${ }^{12-14}$ The estimated MWD enables to qualitatively describe the significant characteristics of polymer systems, i.e. static moments, polydispersity factor, etc. ${ }^{13,14}$ Alternatively, the MWD features under pulsed irradiation allow in a number of cases to immediately evaluate the rate constants of polymerization $\left(k_{p}\right)$ and a polymer chain termination $\left(k_{t}\right)$ in the same experiment ${ }^{4-6,15-18}$ which is not feasible under polymerization by $\mathrm{CW}$ radiation. Though, the calculation of MWD and, especially, the analysis of MWD dynamics during polymerization involve severe difficulties and require numerical techniques. 
At the present time, several papers ${ }^{2,15,19,20}$ are available that consider the features of MWDs resulting from medium irradiation by pulsed laser. A polymerization scheme was studied that comprised the reactions of polymer chains initiation, propagation and termination by recombination of growing polymer chains or their disproportionation. The MWDs under pseudostationary polymerization ${ }^{15}$ with small degree of monomer-to-polymer conversion were calculated on the assumption that the Flory principle ${ }^{13}$ is satisfied and growing radicals are instantaneously generated. It was shown that under pseudostationary regime of polymerization the equidistant peaks exist in the MWD spectrum, the heights of them falling rapidly with chain length increase. For the same polymerization scheme the MWD was obtained under single-pulse irradiation by method of generating functions, and the MWD dynamics in the polymerization process was studied. ${ }^{19}$ The same problem, but with allowance for inhibition action of radicals resulting from photoinduced decomposition of non-symmetrical initiator by laser pulse, was solved too. ${ }^{20}$

The present paper studies theoretically the MWDs resulting from polymerization initiation by an arbitrary sequence of short laser pulses.

\section{SCHEME AND KINETIC MODEL OF POLYMERIZATION}

Consideration was given to the pulsed-laser-initiated free radical polymerization of monomers (M) having a single reactive double bond. Polymerization of such compounds produces linear macromolecules differing in the number of elementary links (i). It was supposed that with radiation absorption the initiator (I) instantaneously dissociates into two radicals similar in reactivity:

$$
\mathrm{I} \stackrel{\mathrm{hv}}{\longrightarrow} 2 \mathrm{X}
$$

The $\mathrm{X}$ radicals react with monomer $(\mathrm{M})$

$$
\mathrm{X}+\mathrm{M} \stackrel{\mathrm{k}_{\mathrm{p}}}{\longrightarrow} \mathrm{XM}_{1}
$$

and initiate the polymerization reaction

$$
\mathrm{XM}_{\mathrm{i}}+\mathrm{M} \stackrel{\mathrm{k}_{\mathrm{p}}}{\longrightarrow} \mathrm{XM}_{\mathrm{i}+1}
$$

Termination of growing chains may occur as a result of recombination of radicals with rate constant $\mathrm{k}_{\mathrm{t}}^{\mathrm{b}}$

$$
\mathrm{XM}_{\mathrm{j}}+\mathrm{XM}_{\mathrm{i}-\mathrm{j}} \stackrel{\mathrm{k}_{\mathrm{t}}^{\mathrm{b}}}{\longrightarrow} \mathrm{XM}_{\mathrm{i}} \mathrm{X}
$$

and disproportionation reaction with rate constant $k_{t}^{d}$

$$
\mathrm{XM}_{\mathrm{j}}+\mathrm{XM}_{\mathrm{i}} \stackrel{\mathrm{k}_{\mathrm{t}}^{\mathrm{d}}}{\longrightarrow} \mathrm{XM}_{\mathrm{j}} \mathrm{X}+\mathrm{M}_{\mathrm{i}}
$$

At any arbitrary moment $\mathrm{t}>0$, the polymerization products are growing radicals $\mathrm{XM}_{\mathrm{i}}$, "dead" macromolecules $\mathrm{XM}_{\mathrm{i}} \mathrm{X}$ devoid of double bonds, and macromolecules $M_{i}$ having a reactive group and capable of participating in polymerization reaction. 
Though, as a probability of $\mathbf{M}_{\mathbf{i}}$ involvement in the chain propagation reaction (3) is slight at the small degree of monomer-to-polymer conversion, $\mathbf{M}_{\mathbf{i}}$ were regarded as dead polymer. It was supposed that the Flory principle ${ }^{13}$ was satisfied, whereby the rate constants of propagation $k_{p}$ and those of chain termination $k_{t}^{b}$ and $k_{t}^{d}$ are not functions of chain length $i$. An option to simplification of the model is equality of rate constants of initiation (2) and chain propagation (3) reactions.

The discussed polymerization model was described by the following set of kinetic equations for initiating radicals concentrations $[\mathrm{X}]=\mathrm{A}_{0}$, growing radicals $\left[\mathrm{XM}_{\mathrm{i}}\right]=A_{\mathrm{i}}$ and dead macromolecules $\left[\mathrm{XM}_{\mathrm{i}} \mathrm{X}\right]+\left[\mathrm{M}_{\mathrm{i}}\right]=\mathrm{B}_{\mathrm{i}}$ :

$$
\begin{aligned}
& \frac{\mathrm{dA}_{0}}{\mathrm{dt}}=-\mathrm{k}_{\mathrm{p}} \mathrm{A}_{0}[M]-\mathrm{k}_{\mathrm{t}} \mathrm{A}_{0} \sum_{j=0}^{\infty} \mathrm{A}_{\mathrm{j}}, \\
& \frac{\mathrm{dA}_{\mathrm{i}}}{\mathrm{dt}}=\mathrm{k}_{\mathrm{p}} \mathrm{A}_{\mathrm{i}-1}[\mathrm{M}]-\mathrm{k}_{\mathrm{p}} \mathrm{A}_{\mathrm{i}}[\mathrm{M}]-\mathrm{k}_{\mathrm{t}} \mathrm{A}_{\mathrm{i}} \sum_{j=0}^{\infty} \mathrm{A}_{\mathrm{j}}, \\
& \frac{\mathrm{dB}}{\mathrm{dt}}=0.5 \mathrm{k}_{\mathrm{t}}^{\mathrm{b}} \sum_{j=0}^{i} \mathrm{~A}_{\mathrm{j}} \mathrm{A}_{\mathrm{i}-\mathrm{j}}+\mathrm{k}_{\mathrm{t}}^{\mathrm{d}} \mathrm{A}_{\mathrm{i}} \sum_{j=0}^{\infty} \mathrm{A}_{\mathrm{j}},
\end{aligned}
$$

where $k_{t}=k_{t}^{b}+k_{t}^{d}$.

\section{GENERAL SOLUTION OF THE SET OF KINETIC EQUATIONS}

Let the initiating radicals concentration $A_{0}^{(1)}=W_{1}$ be produced in the medium at a moment $t=0$ as a result of single-pulse irradiation. The temporal dynamics of growing radicals and dead macromolecules distribution along the chain length on termination of chains by radicals recombination is already known from. ${ }^{10}$ With these results it can be shown that if chain termination by disproportionation is allowed for too, time dependencies of $A_{i}^{(1)}$ and $B_{i}^{(1)}$ have the form:

$$
\begin{aligned}
A_{i}^{(1)}= & W_{1}\left(1+V_{1} t\right)^{-1} \frac{\left(U_{t}\right)^{i} \exp (-U t)}{i !} \\
B_{i}^{(1)}= & 0.5 k_{t}^{b} W_{1}^{2} \int_{0}^{t}\left(1+V_{1} p\right)^{-2} \frac{(2 U p)^{i} \exp (-2 U p)}{i !} d p \\
& +k_{t}^{d} W_{1}^{2} \int_{0}^{t}\left(1+V_{1} p\right)^{-2} \frac{(U p)^{i} \exp (-U p)}{i !} d p
\end{aligned}
$$

where $\mathrm{U}=\mathrm{k}_{\mathrm{p}}[\mathrm{M}]$ and $\mathrm{V}_{1}=\mathrm{k}_{\mathrm{t}} \mathrm{W}_{1}$.

Let the initiating radicals with $\mathrm{W}_{2}$ concentration be generated in the medium at $T_{1}$ interval after the first pulse. Shift now the time origin to the moment of these radicals appearance. Then, for the initial concentration of radicals $A_{i}^{(2)}$ we can write:

$$
\mathrm{A}_{\mathrm{i}}^{(2)}=\mathrm{W}_{2} \delta_{0, \mathrm{i}}+\mathrm{W}_{1}\left(1+\mathrm{V}_{1} \mathrm{~T}_{1}\right)^{-1} \frac{\left(\mathrm{UT}_{1}\right)^{\mathrm{i}} \exp \left(-\mathrm{UT}_{1}\right)}{\mathrm{i} !}
$$


Function

$$
\mathrm{F}_{\mathbf{Z}}^{(2)}=\sum_{\mathrm{i}=0}^{\infty} \mathbf{Z}^{\mathrm{i}} \mathbf{A}_{\mathrm{i}}^{(2)}
$$

was used as a generating one. With $Z=1$ eq. (12) gives a summary concentration of radicals in the polymerization medium after the action of two laser pulses. As the kinetic equations (6)-(8) are valid for $A_{i}^{(2)}$ as well, then, differentiating (12), we obtain

$$
\frac{\mathrm{dF}_{\mathrm{z}}^{(2)}}{\mathrm{dt}}=\mathrm{ZUF}_{\mathrm{z}}^{(2)}-\mathrm{UF}_{\mathrm{z}}^{(2)}-\mathrm{k}_{\mathrm{t}} \mathrm{F}_{\mathrm{z}}^{(2)} \mathrm{F}_{1}^{(2)}
$$

When integrating (13) at $\mathrm{Z}=1$, we find that

$$
\mathrm{F}_{1}^{(2)}=\frac{\mathrm{F}_{1}^{(2)}(0)}{1+\mathrm{k}_{\mathrm{t}} \mathrm{F}_{1}^{(2)}(0) \mathrm{t}}=\frac{\mathrm{F}_{1}^{(2)}(0)}{1+\mathrm{V}_{2} \mathrm{t}}
$$

and, allowing for (11) and (12), obtain

$$
\begin{aligned}
F_{1}^{(2)}(0) & =\sum_{i=0}^{\infty} A_{i}^{(2)}(0)=W_{2}+W_{1}\left(1+V_{1} T_{1}\right)^{-1} \\
V_{2} & =k_{t} W_{2}+V_{1}\left(1+V_{1} T_{1}\right)^{-1}
\end{aligned}
$$

The general solution of (13) has the form

$$
F_{z}^{(2)}=F_{z}^{(2)}(0)\left(1+V_{2} t\right)^{-1} \exp (-U t) \exp (-Z U t)
$$

where

$$
F_{z}^{(2)}(0)=W_{2}+W_{1}\left(1+V_{1} T_{1}\right)^{-1} \sum_{j=0}^{\infty} Z^{j} \frac{\left(U_{1}\right)^{i} \exp \left(-U T_{1}\right)}{j !}
$$

Relying on the expansion

$$
\exp (-U Z t)=\sum_{k=0}^{\infty} Z^{k} \frac{(U t)^{k}}{k !}
$$

and regarding (18) $\mathrm{F}_{\mathbf{Z}}^{(2)}$ can be represented as

$$
\begin{aligned}
F_{z}^{(2)}= & \left(1+V_{2} t\right)^{-1}\left[W_{2} \sum_{i=0}^{\infty} Z^{i} \frac{\left(U T_{1}\right)^{i} \exp \left(-U T_{1}\right)}{i !}\right. \\
& \left.+W_{1}\left(1+V_{1} T_{1}\right)^{-1} \exp \left(-U\left(T_{1}+t\right)\right) \sum_{k, j=0}^{\infty} Z^{k+j} \frac{\left(U T_{1}\right)^{j}(U t)^{k}}{j ! k !}\right]
\end{aligned}
$$


As

$$
\sum_{k, j=0}^{\infty} Z^{k+j} \frac{\left(U T_{1}\right)^{j}(U t)^{k}}{j ! k !}=\sum_{i=0}^{\infty} Z^{i} \frac{\left(U\left(T_{1}+t\right)\right)^{i}}{i !}
$$

then

$$
\begin{aligned}
F_{Z}^{(2)}= & \sum_{i=0}^{\infty} Z^{i}\left(1+V_{2} t\right)^{-1}\left[W_{2} \frac{\left(U_{1}\right)^{i} \exp \left(-U_{1}\right)}{i !}\right. \\
& \left.+W_{1}\left(1+V_{1} T_{1}\right)^{-1} \frac{\left(U\left(T_{1}+t\right)\right)^{i} \exp \left(-U\left(T_{1}+t\right)\right)}{i !}\right]
\end{aligned}
$$

Comparing this expression with (12) for distribution of growing radicals concentrations, we obtain:

$$
\begin{aligned}
A_{i}^{(2)}= & \left(1+V_{2} t\right)^{-1}\left[W_{2} \frac{\left(U T_{1}\right)^{i} \exp \left(-U_{1}\right)}{i !}\right. \\
& \left.+W_{1}\left(1+V_{1} T_{1}\right)^{-1} \frac{\left(U\left(T_{1}+t\right)\right)^{i} \exp \left(-U\left(T_{1}+t\right)\right)}{i !}\right]
\end{aligned}
$$

Distribution of dead macromolecules concentrations is found by integrating (8). With allowance for (14) and (21), we obtain:

$$
\begin{aligned}
\mathrm{B}_{\mathrm{i}}^{(2)}=\mathrm{B}_{\mathrm{i}}^{(1)}\left(\mathrm{T}_{1}\right)+0.5 \mathrm{k}_{\mathrm{t}}^{\mathrm{b}} \int_{0}^{\mathrm{t}}\left(1+\mathrm{V}_{2} \mathrm{p}\right)^{-2}\left[\mathrm{~W}_{2}^{2} \frac{(2 \mathrm{Up})^{\mathrm{i}} \exp (-2 \mathrm{Up})}{\mathrm{i} !}\right. \\
+2 \mathrm{~W}_{1} \mathrm{~W}_{2}\left(1+\mathrm{V}_{1} \mathrm{~T}_{1}\right)^{-1} \frac{\left(\mathrm{U}\left(\mathrm{T}_{1}+2 \mathrm{p}\right)\right)^{\mathrm{i}} \exp \left(-\mathrm{U}\left(\mathrm{T}_{1}+2 \mathrm{p}\right)\right)}{\mathrm{i} !} \\
\left.+\mathrm{W}_{1}^{2}\left(1+\mathrm{V}_{1} \mathrm{~T}_{1}\right)^{-2} \frac{\left(2 \mathrm{U}\left(\mathrm{T}_{1}+\mathrm{p}\right)\right)^{\mathrm{i}} \exp \left(-2 \mathrm{U}\left(\mathrm{T}_{1}+\mathrm{p}\right)\right)}{\mathrm{i} !}\right] \mathrm{dp} \\
+\frac{\mathrm{k}_{\mathrm{t}}^{\mathrm{b}}}{\mathrm{k}_{\mathrm{t}}} \int_{0}^{\mathrm{t}} \mathrm{V}_{1}\left(1+\mathrm{V}_{2} \mathrm{p}\right)^{-2}\left[\mathrm{~W}_{2} \frac{(\mathrm{Up})^{\mathrm{i}} \exp (-\mathrm{Up})}{\mathrm{i} !}\right. \\
\left.+\mathrm{W}_{1}\left(1+\mathrm{V}_{1} \mathrm{~T}_{1}\right)^{-1} \frac{\left(\mathrm{U}\left(\mathrm{T}_{1}+\mathrm{p}\right)\right)^{\mathrm{i}} \exp \left(-\mathrm{U}\left(\mathrm{T}_{1}+\mathrm{p}\right)\right)}{\mathrm{i} !}\right] \mathrm{dp} .
\end{aligned}
$$

Performing the same mathematics, as used in calculating $\mathrm{A}_{\mathrm{i}}^{(2)}$ and $\mathrm{B}_{\mathrm{i}}^{(2)}$ distributions after the $\mathrm{N}$-pulse that produces initiating radicals with $\mathrm{W}_{\mathbf{N}}$ concentration in the 
medium, we derive:

$$
\begin{aligned}
A_{i}^{(N)} & =\left(1+V_{N} t\right)^{-1} \sum_{j=1}^{N} S_{j}^{N} \frac{\left(U\left(Q_{j}^{N}+t\right)\right)^{i} \exp \left(-U\left(Q_{j}^{N}+t\right)\right)}{i !} \\
B_{i}^{(N)} & =0.5 k_{t}^{b} \sum_{k=1}^{N-1} \int_{0}^{T_{k}}\left(1+V_{k} p\right)^{-2} \times \\
& \times \sum_{j, m=1}^{K} S_{m}^{k} S_{j}^{k} \frac{\left(U\left(Q_{m}^{k}+Q_{j}^{k}+2 p\right)\right)^{i} \exp \left(-U\left(Q_{m}^{k}+Q_{j}^{k}+2 p\right)\right)}{i !} d p \\
& +0.5 k_{t}^{b} \int_{0}^{t}\left(1+V_{N} p\right)^{-2} \times \\
& \times \sum_{j, m=1}^{N} S_{m}^{N} S_{j}^{N} \frac{\left(U\left(Q_{m}^{N}+Q_{j}^{N}+2 p\right)\right)^{i} \exp \left(-U\left(Q_{m}^{N}+Q_{j}^{N}+2 p\right)\right)}{i !} d p \\
& +\frac{k_{t}^{d} k_{t}^{N}-1}{\sum_{k=1}} \int_{0}^{T_{k}} V_{k}\left(1+V_{k} p\right)^{-2} \sum_{j=1}^{k} S_{j}^{k} \frac{\left(U\left(Q_{j}^{k}+p\right)\right)^{i} \exp \left(-U\left(Q_{j}^{k}+p\right)\right)}{i !} d p \\
& +\frac{k_{t}^{d}}{k_{t}} \int_{0}^{t} V_{N}\left(1+V_{N} p\right)^{-2} \sum_{j=1}^{N} S_{j}^{N} \frac{\left(U\left(Q_{j}^{N}+p\right)\right)^{i} \exp \left(-U\left(Q_{j}^{N}+p\right)\right)}{i !} d p+
\end{aligned}
$$

where

$$
\begin{aligned}
& \mathrm{Q}_{\mathrm{j}}^{\mathrm{k}}=\sum_{\mathrm{r}=1}^{\mathrm{j}-1} \mathrm{~T}_{\mathrm{k}-\mathrm{r}} \\
& \mathrm{V}_{\mathrm{k}}=\mathrm{k}_{\mathrm{t}} \mathrm{W}_{\mathrm{k}}+\mathrm{V}_{\mathrm{k}-1}\left(1+\mathrm{V}_{\mathrm{k}-1} \mathrm{~T}_{\mathrm{k}-1}\right)^{-1}, \mathrm{~V}_{1}=\mathrm{k}_{\mathrm{t}} \mathrm{W}_{1}, \mathrm{k}=2,3, \ldots, \mathrm{N}-1, \mathrm{~N} \\
& \mathrm{~S}_{\mathrm{j}}^{\mathrm{k}}=\mathrm{W}_{\mathrm{k}-\mathrm{j}+1} \prod_{\mathrm{r}=1}^{\mathrm{j}-1}\left(1+\mathrm{V}_{\mathrm{k}-\mathrm{r}} \mathrm{T}_{\mathrm{k}-\mathrm{r}}\right)^{-1}
\end{aligned}
$$

Thus, on medium irradiation, with an arbitrary sequence of $\mathrm{N}$ pulses at any moment $t$ after the $\mathrm{N}$ pulse, distribution of growing radicals comprises a sum of $\mathrm{N}$ particle groups having Poisson distribution. Coefficients $S_{j}^{k}$ present the remaining by the moment of $k$-pulse concentration of radicals produced by $(k-j+1)$ pulse. $Q_{j}^{k}$ is the time interval between the $(\mathrm{k}-\mathrm{j}+1)$ and $\mathrm{k}$ pulses. There exists a simple relation between $V_{k}$ parameters and summary concentration of radicals $F_{1}^{(k)}(0)$ immediately after the $\mathrm{k}$ pulse:

$$
\mathrm{V}_{\mathrm{k}}=\mathrm{k}_{\mathrm{t}} \mathrm{F}_{1}^{(\mathrm{k})}(0)
$$

The degree of monomer-to-polymer conversion can be determined by integrating

$$
\frac{d[M]}{d t}=-k_{p}[M] \sum_{j=0}^{\infty} A_{j},
$$


With small degree of monomer-to-polymer conversion after the N-pulse, concentration of monomer $\left[\mathrm{M}^{(\mathbf{N})}\right]$ is

$$
\begin{aligned}
{\left[M^{(N)}\right] } & =\left[M^{(N-1)}\right]-\frac{U}{k_{t}} \ln \left(1+V_{N} t\right) \\
& =\left[M^{(0)}\right]-\frac{U}{k_{t}} \sum_{i=1}^{N-1} \ln \left(1+V_{i} T_{i}\right)-\frac{U}{k_{t}} \ln \left(1+V_{N} t\right) .
\end{aligned}
$$

This expression was used to verify whether the degree of monomer-to-polymer conversion is small.

\section{CALCULATION RESULTS AND DISCUSSION}

The derived above expressions for $A_{i}^{(N)}$ and $B_{i}^{(N)}$ were used in the study of distributions produced by pulse-periodic irradiation and by irradiation with a periodic sequence of pulses packets. Before the calculation results are cited, we shall consider the question of the transition period duration, i.e. the time of polymerization pseudostationary regime establishment. This regime features a periodic variation in the summary concentration of radicals $(R(t))$ with frequency equal to pulse repetition rate. ${ }^{15} \mathrm{By}$ this, the values of maximum and minimum radicals concentration does not vary with time.

\section{Pseudostationary Polymerization Attainment}

As the radicals concentration after the $N$-pulse action is $F_{1}(0)$, then with pulseperiodical irradiation the $\mathrm{R}(\mathrm{t})$ relationship represents a piece-smooth function

$$
R(t)=F_{1}^{(N)}(0)\left(1+V_{N} t\right)^{-1}
$$

where $(\mathrm{N}-1) \mathrm{T} \leqslant \mathrm{t} \leqslant \mathrm{NT}, \mathrm{N}=1,2, \ldots, \mathrm{T}=\mathrm{f}^{-1}, \mathrm{~F}_{1}(0)$ and $\mathrm{V}_{\mathrm{N}}$ are correlated through $(30)$, and $V_{N}$ are found with (28).

Figure 1 depicts time dependencies of $\mathrm{R}(\mathrm{t}) / \mathrm{W}$ (W is radicals concentration immediately generated by a single laser pulse), calculated for $\mathrm{k}_{\mathrm{t}}=10^{6}, 10^{7}, 10^{8}$ $1 /(\mathrm{mol} \cdot \mathrm{s})$ for $\mathrm{W}=10^{-7} \mathrm{~mol} / 1$ and $\mathrm{T}=0.2 \mathrm{~s}$. With $\mathrm{k}_{\mathrm{t}}=10^{6} 1 /(\mathrm{mol} \cdot \mathrm{s})\left(\mathrm{k}_{\mathrm{t}} \mathrm{WT}>1\right)$, pseudostationary regime of polymerization is set just following the second pulse. For lesser values of $k_{t}$ satisfying the $k_{t} W T<1$ condition, this regime is attained only following a number of pulses $\mathrm{N}^{\mathrm{ps}}$. If $\mathrm{k}_{\mathrm{t}}=10^{6} 1 /(\mathrm{mol} \cdot \mathrm{s})$, the amplitude of radicals concentration vibrations accounts for $\mathrm{W}$ and is much lower than average value of $R$. The reduction of $k_{t} W T$ causes the decrease in vibration amplitude, and polymerization regime approaches the quasi-stationary state. With quasi-stationary polymerization, the initiation velocity $v_{\text {in }}$ is equal to that of chain termination $v_{t}$. So, for 


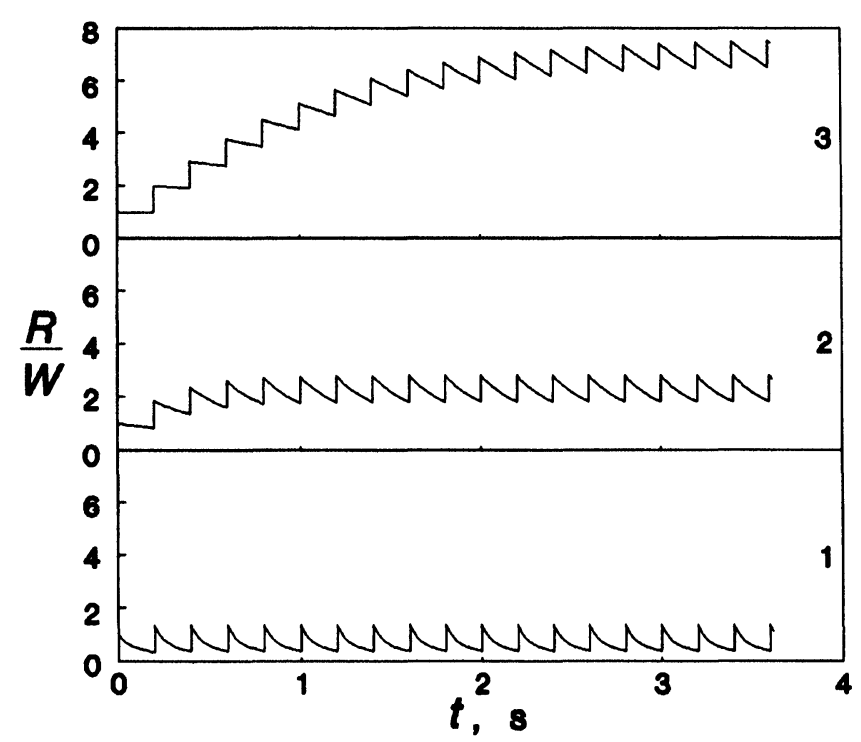

Figure 1 Dependencies of relative concentrations of radicals $R / W$ on time $t$. Calculated for $k_{t}=10^{6}(1)$, $10^{7}(2), 10^{8}(3) 1 /(\mathrm{mol} \cdot \mathrm{s}), \mathrm{W}=10^{-7} \mathrm{~mol} / 1$ and $\mathrm{T}=0.2 \mathrm{~s}$.

the average concentration of radicals $R_{a v}$ under

$$
\mathrm{v}_{\mathrm{t}} \approx \mathrm{k}_{\mathrm{t}} \mathrm{R}_{\mathrm{av}}^{2} \approx \mathrm{v}_{\mathrm{in}} \approx \mathrm{W} / \mathrm{T}
$$

Then,

$$
\mathrm{R}_{\mathrm{av}} \approx\left(\mathrm{W} / \mathrm{k}_{\mathrm{t}} \mathrm{T}\right)^{1 / 2}
$$

If $\mathrm{k}_{\mathrm{t}} \mathrm{W} \mathrm{T} \ll 1$, this expression agrees with that for maximum concentration of radicals $\mathrm{R}_{\mathrm{m}}^{\mathrm{ps}}$ under pseudostationary polymerization ${ }^{14}$.

$$
\mathrm{R}_{\mathrm{m}}^{\mathrm{ps}}=0.5 \mathrm{~W}+0.5 \mathrm{~W}\left(1+4 / \mathrm{k}_{\mathrm{t}} \mathrm{W} \mathrm{T}\right)^{1 / 2}
$$

Fulfillment of the condition

$$
\frac{\mathrm{R}_{\mathrm{m}}^{\mathrm{ps}}-\mathrm{F}_{1}^{(\mathrm{N})}(0)}{\mathrm{R}_{\mathrm{m}}^{\mathrm{ps}}} \leqslant \varepsilon
$$

can serve as a criterion for attainment of pseudostationary regime of polymerization. Figure 2 shows the $k_{t} W$ T-dependence of the number of pulses $N_{\varepsilon}^{\text {ps }}$ required for establishment of pseudostationary regime in the medium. The relation has been calculated for $\varepsilon=10^{-3}$. For small $\mathrm{k}_{\mathrm{t}} \mathrm{WT}, \mathrm{k}_{\mathrm{t}} \mathrm{WT}$-dependence of $\mathrm{N}_{\varepsilon}^{\mathrm{ps}}$ is well described by the expression

$$
\mathrm{N}_{\varepsilon}^{\mathrm{ps}} \approx \mathrm{a}_{\varepsilon} /\left(\mathrm{k}_{\mathrm{t}} \mathrm{WT}\right)^{1 / 2}
$$

where $\mathrm{a}_{\varepsilon}$ is the $\varepsilon$-dependent coefficient. For example, at $\varepsilon=0.1 \mathrm{a}_{\varepsilon} \approx 1.5$, at $\varepsilon=0.01$ $\mathrm{a}_{\varepsilon} \approx 2.7$ and at $\varepsilon=0.001 \mathrm{a}_{\varepsilon} \approx 3.8$. 


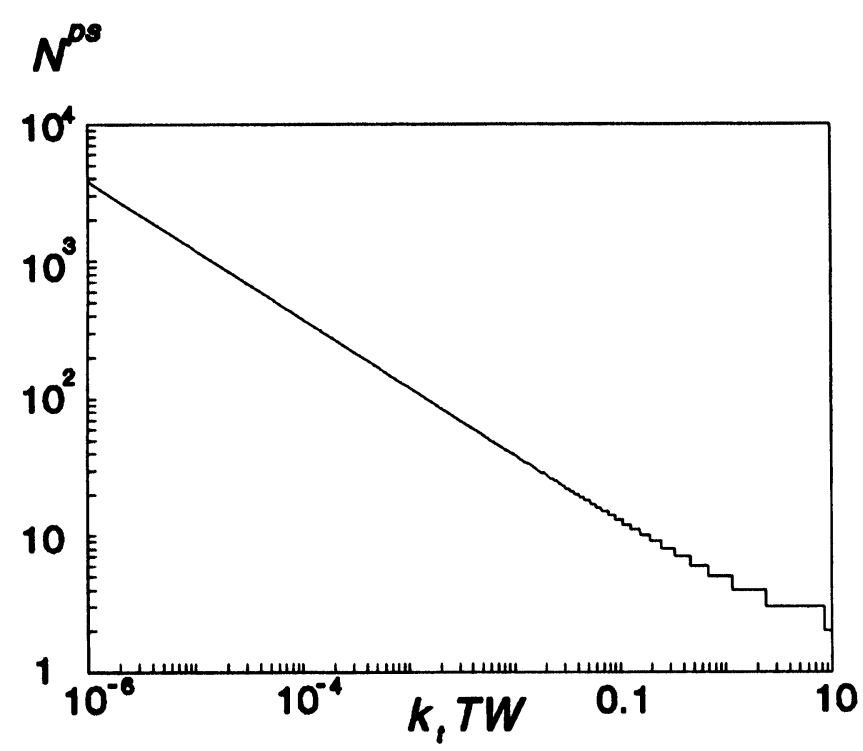

Figure $2 k_{t}$ WT-dependence of the number of laser pulses $N_{\varepsilon}^{p s}$ required to attain pseudostationary regime of polymerization.

Thus, with pulse-periodical irradiation, duration of the transition period runs $\mathrm{TN}_{\varepsilon}^{\text {ps. }}$. Through this time, leveling of concentrations takes place, which are initiated and disappear within one period as a result of radicals termination; average concentration of radicals and, consequently, average polymerization rate are increased.

\section{MWD Dynamics on Pulse-Periodical Initiation}

Figure 3 presents the distributions of concentrations of growing radicals $A_{i}$ (Figure. 3a) and dead polymer molecules $B_{i}$ (Figure $3 b$ ) calculated for $t=0.1 \mathrm{~s}$ after the considered $\mathrm{N}$-pulse for the case of chain termination by recombination. The distributions were obtained for $\mathrm{U}=300 \mathrm{~s}^{-1}, \mathrm{~T}=0.2 \mathrm{~s}, \mathrm{k}_{\mathrm{t}}=10^{7} 1 /(\mathrm{mol} \cdot \mathrm{s})$ and $\mathrm{W}=10^{-8} \mathrm{~mol} / 1$. As $\mathrm{k}_{\mathrm{t}} \mathrm{WT}=0.02$, then, in accordance with $(38), \mathrm{N}_{\varepsilon}^{\mathrm{ps}} \approx 20$ for $\varepsilon=0.01$. So the distributions generated after $N=1,5$ and 10 pulses can be referred to the period of pseudostationary polymerization attainment, and those after $\mathrm{N}=25$ and 50 pulses involve the case when this regime has already been achieved.

The $A_{i}$ spectra present a aggregate of radical groups, each having Poisson distribution. A radical group with the shortest chain lengths $i$ (the first one in the spectrum) corresponds to the last pulse of $\mathrm{N}$-sequence. With time, the number of radicals in the group reduces, the group maximum shifts to large i-values $(\sim t)$ and the group width increases $\left(\sim t^{1 / 2}\right)$. So, at large $N$ (for $N=25$ and 50 in Figure 3a) only groups produced by the last pulses show up in $A_{i}$ spectra. The radical groups generated by earlier pulses shift to the region of large $i$ and broaden, thus forming the continuous spectrum. 

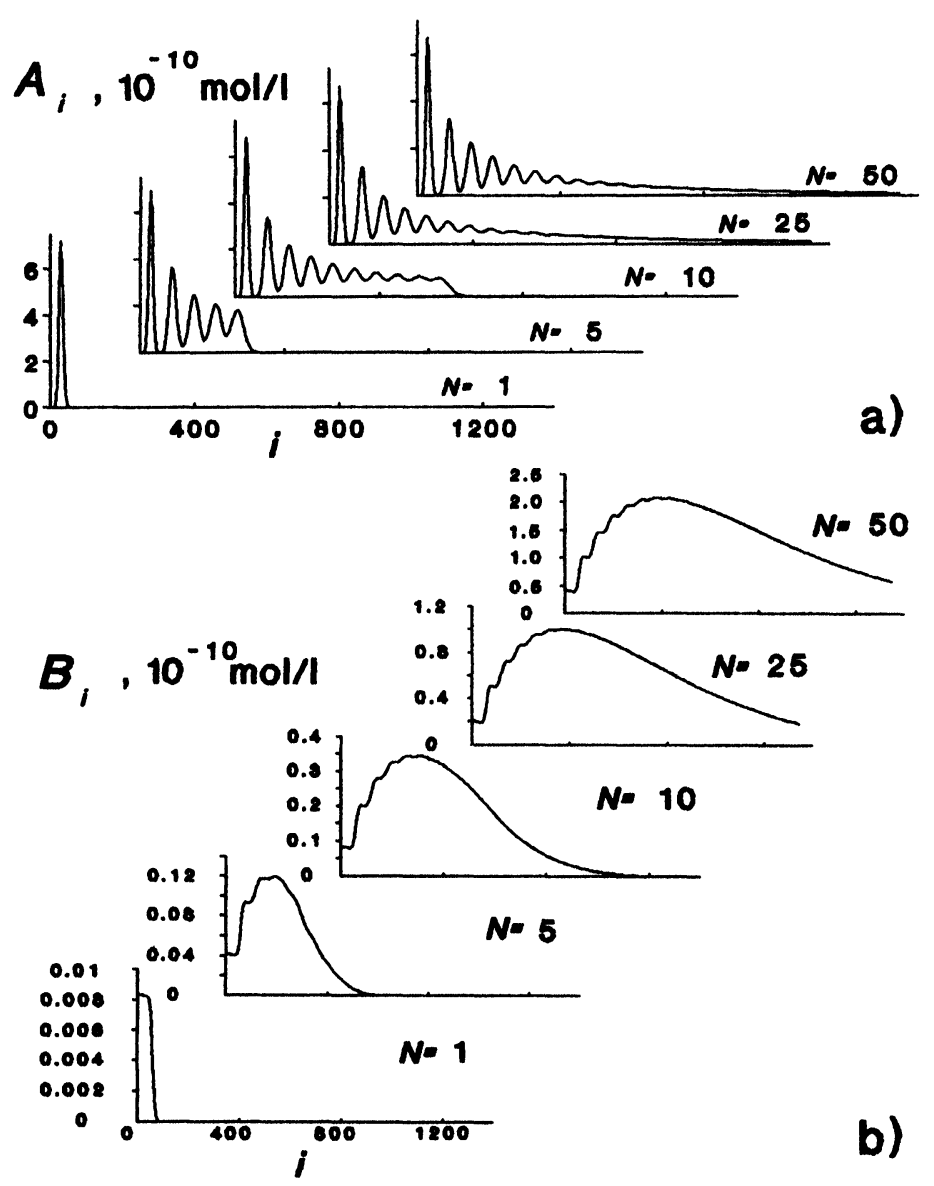

Figure 3 Distributions of concentrations of growing $A_{i}(a)$ and dead $B_{i}(b)$ macromolecules along chain lengths $\mathrm{i}$ in $0.1 \mathrm{~s}$ after the $\mathrm{N}$-pulse for the case of chain termination by recombination of radicals. Calculated for $\mathrm{k}_{\mathrm{p}}[\mathrm{M}]=300 \mathrm{~s}^{-1}, \mathrm{k}_{\mathrm{t}}=10^{7} 1 /(\mathrm{mol} \mathrm{s})$ and $\mathrm{W}=10^{-8} \mathrm{~mol} / 1$ and $\mathrm{T}=0.2 \mathrm{~s}$.

The reduction of radicals number in the group is due both to recombination of them with each other and, more substantially, to their interaction with the radicals of other groups, the summary concentration of which increases within the period of pseudostationary state attainment. As follows from (35) and (38), the effective lifetime of radicals approximates $N_{\varepsilon}^{p s} T$ and the pattern of $A_{i}$ distribution is governed mainly by interaction of radical groups produced by the last $N_{\varepsilon}^{p s}$ pulses. The radicals spectrum in the pseudostationary state is a periodic function of $t\left(A_{i}(t) \approx A_{i}(t+T)\right)$.

The distribution of dead macromolecules along the chain length varies significantly as well at the period of pseudostationary state attainment and slightly changes after this regime has been established (Figure $3 b$ ). The abrupt termination of $\mathbf{B}_{i}$ spectrum at $\mathrm{N}=1$ depends upon the fact that it has been calculated for $t=0.1 \mathrm{~s}$, and by this moment only macromolecules with chain length $i \leqslant 2 \mathrm{Ut}$ have managed to 
appear. At the period of pseudostationary regime establishment, the general maximum of $B_{i}$ distribution is shifted to large $i$-values with $N$ growth, and the width of distribution increases. The abrupt increase in dead macromolecules concentrations with $\mathrm{i} \approx \mathrm{k}_{\mathrm{p}}[\mathrm{M}] \operatorname{Tn}(\mathrm{n} \leqslant \mathrm{N}-1)$ is a consequence of recombination of a radical group produced by the last pulse with those generated by earlier pulses. As by the moment of the $\mathrm{N}$-pulse onset the concentration of radicals produced by the $(\mathrm{N}-1)$ pulse is the largest, then the contribution of these radical groups recombination to a dead polymer formation is the greatest. In the pseudostationary state, $\mathrm{N}$ growth is responsible for the linear increase in the summary number of dead macromolecules (by $\approx \mathrm{W} / 2$ after each pulse). In this case, location of $\mathrm{B}_{\mathrm{i}}$ maximum and the distribution pattern do not practically change $\left(B_{i}^{(N)}(t) / N \approx B_{i}{ }^{(N+1)}(t+T) /(N+1)\right)$.

At the period of pseudostationary state attainment, the major contribution to the summary distribution $A_{i}+B_{i}$ is made by growing radicals $(N=1,5,10)$. By the moment of the regime establishment, the concentration of dead macromolecules turns essential $(\mathrm{N}=25)$; with $\mathrm{N}$ further increase this contribution will grow and the portion of growing radicals will reduce.

Let us consider now in greater detail the effect of parameters of initiating pulses ( $\mathrm{T}$ and $\mathrm{W}$ ) and polymerizing medium $\left(\mathrm{k}_{\mathrm{p}}\right.$ and $\left.\mathrm{k}_{\mathrm{t}}\right)$ upon MWD.

\section{Influence of Irradiation Period upon MWD Pattern}

Figure 4 depicts $B_{i}$ distributions produced at the moment $t=30 \mathrm{~s}$ following the last pulse on polymerization initiated by a sequence of 50 pulses. The calculation was performed for the case of chain termination by radicals recombination for $\mathrm{U}=\mathrm{k}_{\mathrm{p}}[\mathrm{M}]=300 \mathrm{~s}^{-1}, \mathrm{k}_{\mathrm{t}}=10^{7} 1 /(\mathrm{mol} \cdot \mathrm{s})$ and $\mathrm{W}=10^{-8} \mathrm{~mol} / 1$. With this parameters and $\varepsilon=10^{-2}$ for $\mathrm{T}=0.01-10 \mathrm{~s}, \mathrm{~N}_{\varepsilon}^{\mathrm{ps}}<50$.

For $\mathrm{T}=0.01 \mathrm{~s}(\mathrm{f}=100 \mathrm{~Hz})$ in dead macromolecules distribution, pulse-periodical nature of irradiation does not exhibit itself. The dashed line in Figure 4 illustrates the distributions generated in the case of quasi-stationary polymerization with initiating rate $v_{\text {in }}=W / T$. These distributions were calculated with the formula

$$
B_{i}^{q}=\frac{k_{t}^{b} v_{i n}^{2} a^{i}(i+1) t}{2\left(U+k_{t}^{b} R_{a v}\right)^{2}}
$$

where $\alpha=U /\left(U+k_{t} R_{a v}\right), R_{a v}$ was found by formula (35), $t=T N$. The $B_{i}$ distribution for $\mathrm{N}=50$ and $\mathrm{T}=0.01$ differs little from the distribution $\mathrm{B}_{\mathrm{i}}^{\mathrm{q}}$ generated under quasistationary polymerization. The increase in $\mathrm{N}$ reduces the distinction between them (Figure 6a). It follows from (39) that

$$
\mathrm{i}_{\max }=(-1 \mathrm{na})^{-1}-1
$$

where $\alpha=\mathrm{U} /\left[\mathrm{U}+\left(\mathrm{k}_{\mathrm{t}} \mathrm{W} / \mathrm{T}\right)^{1 / 2}\right]$, and with $\mathrm{i}_{\max } \gg 1$ and $\mathrm{U} \gg\left(\mathrm{k}_{\mathrm{t}} \mathrm{W} / \mathrm{T}\right)^{1 / 2}, \mathrm{i}_{\max } \approx$ $\left.\mathrm{U}\left(\mathrm{k}_{\mathrm{t}} \mathrm{W} / \mathrm{T}\right)^{1 / 2}\right]$.

Thus, the analysis of distributions produced by pulse-periodical irradiation with high pulse repetition rate is evidence that the formulae to calculate the distributions under pulsed irradiation, reported in this work, can be used to study the 
distributions produced by continuous irradiation; for example, in calculating of MWD in the case that the condition of quasi-stationarity is not met. The relation $\mathrm{UT}=\mathrm{k}_{\mathrm{p}}[\mathrm{M}] \mathrm{T}=10$ approximately determines the boundary separating the region where pulse-periodical irradiation has practically no effect upon the MWD pattern $(\mathrm{UT}<10)$ from that with the strong effect of this kind $(\mathrm{UT}>10)$. This relation was found from the condition of absence of local extremums in $B_{i}$ distribution.

In the case of large $T$ values favoring the fulfillment of the $\mathrm{k}_{\mathrm{t}} \mathrm{WT} \gg 1$ condition, the spectrum $B_{i}$ features a comparitively small number of peaks, the amplitudes of which decrease with $i$ growth (Figure 4 for $T=10 \mathrm{~s}$ ). Here, the pseudostationary regime of polymerization is virtually attained after the second pulse. The distance between maxima, as well as between minima in $B_{i}$ spectrum is

$$
\Delta \mathrm{i}_{\max } \approx \Delta \mathrm{i}_{\min } \approx \mathrm{UT}=\mathrm{k}_{\mathrm{p}}[\mathrm{M}] \mathrm{T}
$$
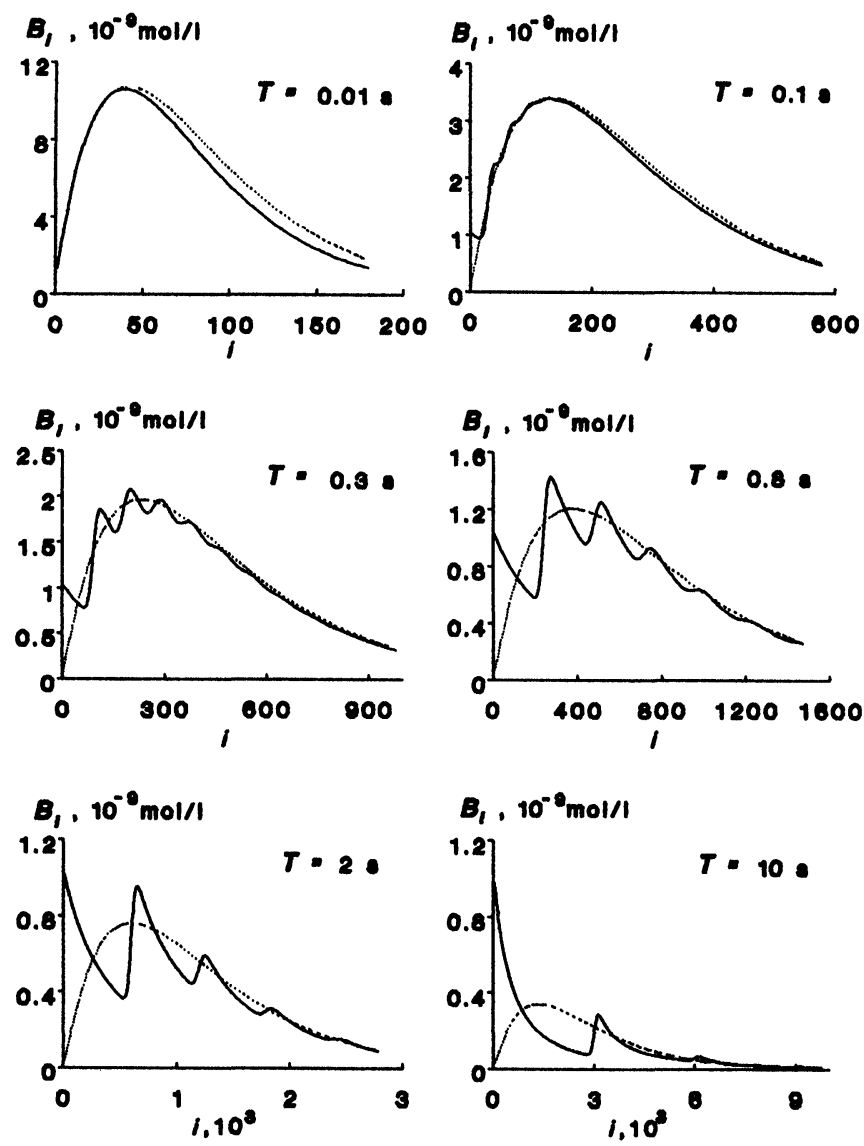

Figure 4 Distribution of $B_{i}$ concentrations of dead macromolecules along chain lengths $i$ in $20 \mathrm{~s}$ after the 50th pulse for different irradiation periods $\mathrm{T}$ in the case of chain termination by recombination of radicals. Calculated for $\mathrm{k}_{\mathrm{p}}[\mathrm{M}]=300 \mathrm{~s}^{-1}, \mathrm{k}_{\mathrm{t}}=10^{7} 1 /(\mathrm{mol} \cdot \mathrm{s})$ and $\mathrm{W}=5.10^{-8} \mathrm{~mol} / \mathrm{l}$. The dashed line illustrates the distributions for quasi-stationary regime of polymerization (see the text). 
In ${ }^{15}$ this simple relation was first used to calculate the absolute value of the rate constant of chain propagation $\mathrm{k}_{\mathrm{p}}$ on polymerization of styrene. It should be noted that if $T<10 /\left(k_{p}[M]\right)$ then (41) can not be used because no separate peaks will be visible in $B_{i}$ spectrum.

With $\mathrm{k}_{\mathrm{t}} \mathrm{WT} \leqslant 1$ and $\mathrm{UT}>10$, a structure induced by pulse-periodical nature of polymerization initiation appears in $B_{i}$ spectrum on the background of distribution approximating that under quasi-stationary regime $\left(B_{i}\right.$ distributions for $T \geqslant 0.1 \mathrm{~s}$ in Figure 4). The comparison between the distributions for $\mathrm{T}=0.3,0.8$ and $2.0 \mathrm{~s}$ alludes to the fact that the technique of $\mathrm{k}_{\mathrm{p}}$ evaluation benefits much from polymerization with $\mathrm{k}_{\mathrm{t}} \mathrm{WT} \leqslant 1$. In this case both the number of maxima and the amplitudes of some peaks in $B_{i}$ spectrum exceed essentially those existing with $T=10 \mathrm{~s}$.

\section{Effect of Polymerizing Medium and Radiation Parameters upon MWD}

Constant $k_{p}$ defines peak widths in distributions of growing radicals and dead macromolecules, as it enters into the parameter of Poisson distribution (see(9), (10), (25), (26)) and the distance between peaks (39). The number of peaks distinguishable in $A_{i}$ and $B_{i}$ distributions grows with $k_{p}$, as the distance between the peaks extends faster than their width. By this, both $i_{\text {max }}$ for separate peaks and width of distributions are increased.

The pattern of growing radicals and dead molecules distribution does not change with $\mathrm{k}_{\mathrm{t}}$ and $\mathrm{W}$, if $\mathrm{V}=\mathrm{k}_{\mathrm{t}} \mathrm{WT}=$ const. Reduction of $\mathrm{V}$ extends the period of polymerization pseudostationary regime attainment (Figure 2). The absolute and relative amplitudes of peaks vary with $\mathrm{V}$; therewith, location and width of peaks in spectra $A_{i}$ and $B_{i}$ are retained. With increase in $V$, the relative amplitude of each subsequent peak in $A_{i}$ and $B_{i}$ spectra is lowered with $i$ growth, and the general maximum $B_{i}$ is shifted to lesser i-values.

\section{Chain Termination by Disproportionation}

Figure 5 shows $B_{i}$ distributions for the case of chain termination by disproportionation, calculated for various pulse repetition rates. In calculations, values parameters were used, for which $B_{i}$ distributions presented in Figure 4 were found. All the distributions in Figure 5 correspond to the attained pseudostationary regime of polymerization. With small $\mathrm{T}, \mathrm{B}_{\mathrm{i}}$ has the pattern close to the distribution formed as the result of chain termination by disproportionation under quasi-stationary polymerization. The distributions for quasistationary polymerization were calculated by the formula

$$
B_{i}^{q}=\frac{k_{i}^{d} v_{\text {in }}^{2} a^{i} t}{R_{a v}\left(U+k_{t}^{d} R_{a v}\right)}
$$

where the relevant parameters are determined in the same way as for (39). With increase in $\mathrm{T}$ on the background of monotonically decreasing distribution, the peaks appear, the spacings between them being found from (41). The key regularities of variation in $A_{i}$ and $B_{i}$ with $k_{t}, k_{p}$ and $W$ are the same as in the case of chain 

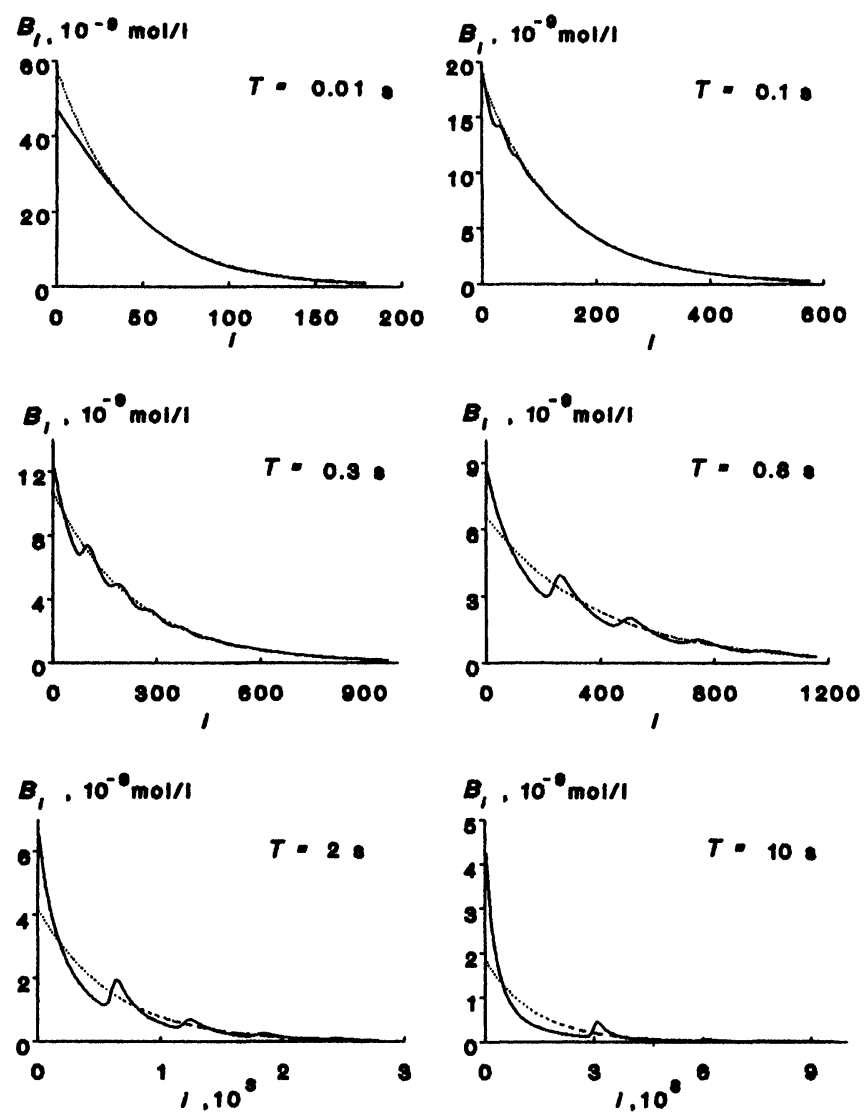

Figure 5 Distributions of $B_{i}$ concentrations of dead macromolecules along chain lengths $i$ in $20 \mathrm{~s}$ after the 50th pulse for different irradiation periods $T$ in the case of chain termination by disproportionation. The parameters of the model are identical to those presented in the caption to Figure 4. The dashed line illustrates the distributions for quasi-stationary regime of polymerization.

termination by recombination. Though it should be noted that in the absence of pseudostationary regime under chain termination by disproportionation, distributions can be produced approximating in pattern those presented in Figure 4 for $k_{t} \mathbf{W} T \leqslant 1$.

\section{MWD on Irradiation with Sequence of Pulses Packets}

At now let's consider the features of distributions generated by polymerization induced with the same number of pulses broken variously down into packets. Here, a case is considered when pulse repetition rate in a packet exceeds much that of the packets on their own.

Figure 6 illustrates the dead macromolecules distributions on polymerization initiated with pulse packets, the spacing between then $t=20 \mathrm{~s}$, for the cases of chain 


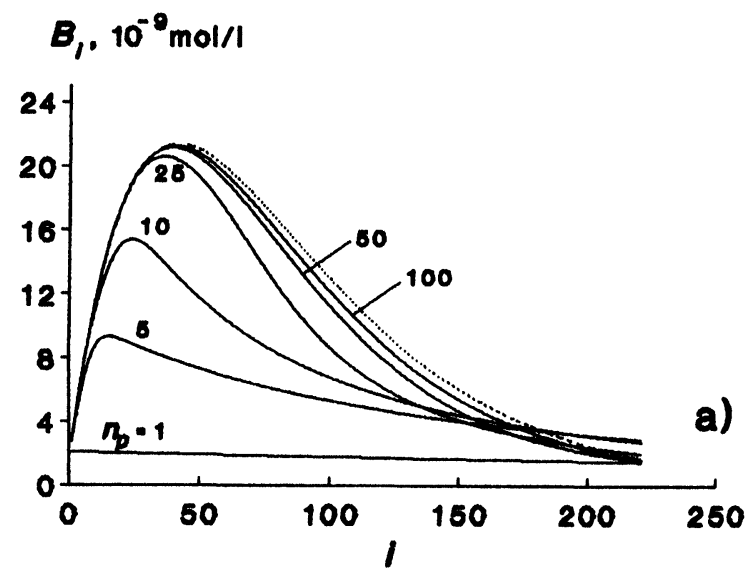

$$
B_{1}, 10^{-8} \mathrm{~mol} / 1
$$

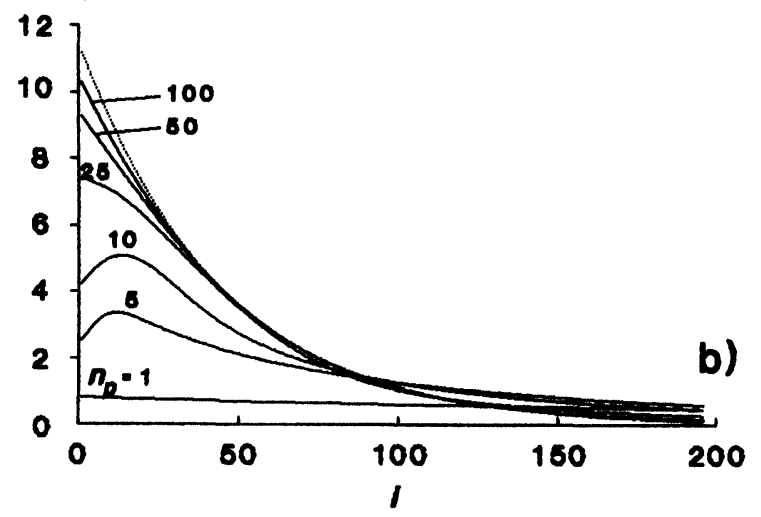

Figure 6 Distributions of $B_{i}$ concentrations of dead macromolecules along chain lengths $i$ on irradiation with 100 pulses broken down into packets of $n_{p}$ pulses for the case of chain termination by recombination of radicals (a) and disproportionation (b). Calculated for the moment $t=20 \mathrm{~s}$ after the last pulse for $\mathrm{k}_{\mathrm{p}}[\mathrm{M}]=300 \mathrm{~s}^{-1}, \mathrm{k}_{\mathrm{t}}=10^{7} 1 /(\mathrm{mol} . \mathrm{s})$ and $\mathrm{W}=5.10^{-8} \mathrm{~mol} / 1$, pulse repetition period in the packet $\mathrm{T}=0.01$ $s$ and packet repetition rate $f=0.05 \mathrm{~Hz}$. The dashed line illustrates the distributions for quasi-stationary regime of polymerization.

termination by recombination (a) and disproportionation (b). For all $\mathbf{B}_{\mathrm{i}}$, pulse repetition rate in the packet $T=0.01 \mathrm{~s}$ and $n_{p} N=100$ (here, $n_{p}$ is the number of pulses in a packet, $\mathrm{N}$ is the number of packets). The rest of the parameters are given in the figure caption. The dashed line shows the distributions calculated by formulae (39) and (42).

With $n_{p}<N_{\varepsilon}^{p s}$ (for $T=0.01 s, N_{\varepsilon}^{p s}=22$ ), $B_{i}$ differ greatly from the distribution formed under quasi-stationary polymerization. In the case of chain termination by recombination (Figure 6a) $i_{\max }$ diminishes with $n_{p}$ and is within the range $\mathrm{n}_{\mathrm{p}} \mathrm{UT} \leqslant \mathrm{i}_{\max } \leqslant \mathrm{i}_{\mathrm{m}} \mathrm{q}\left(\mathrm{i}_{\mathrm{m}} \mathrm{q}\right.$ is a maximum for distribution under quasi-stationary regime of polymerization). With decrease in $n_{p}$, distribution narrowing in $i_{\max }$ region and broadening in the region of large $i$ take place. In the case of chain termination by 
disproportionation, $\mathrm{n}_{\mathrm{p}}$ reduction leads to qualitative change of the distribution itself. A monotonically decreasing distribution is transformed to that typical for chain termination by radicals recombination. The growing radicals with chain length $0<\mathrm{i}<\mathrm{n}_{\mathrm{p}} \mathrm{UT}$ interact for a time $\approx \mathrm{n}_{\mathrm{p}} \mathrm{T}-\mathrm{i} / \mathrm{U}$ with the summary concentration of radicals gradually increasing from $\approx \mathrm{Wi} / \mathrm{TU}$ to $\approx \mathrm{Wn}_{\mathrm{p}}$ (if variation in concentration because of disproportionation reaction is neglected), and for a time $\approx \mathrm{i} / \mathrm{U}$ they interact with concentration $\approx \mathrm{Wn}_{\mathrm{p}}$. This gives rise to maximum origination in distribution $B_{i}$.

\section{CONCLUSIONS}

In the present paper the expressions have been derived that enable to study the distributions of polymer molecules along the chain length on initiation of linear polymerization by an arbitrary sequence of short pulses. These expressions are valid if the constants of chain propagation and termination do not depend on the chain length; the constant of initiation is equal to or much greater than $\mathrm{k}_{\mathrm{p}}$; the degree of monomer-to-polymer conversion is not large $([\mathrm{M}]=$ const $)$ the processes of chain inhibition and transfer are lacking. On these assumptions, a computer investigation of MWD produced on pulse-periodical initiation of polymerization has been undertaken for a broad range of initiating pulses repetition rates and durations. The computing results have brought out that the derived expressions can be applied to study MWD on initiation of polymerization both by arbitrary-duration pulses and by $\mathrm{CW}$ beam.

The research into the temporal dynamics of MWD has disclosed that the pattern of distribution of growing radicals and dead macromolecules greatly varies in the process of pseudostationary state attainment, and under pseudostationary state $\mathrm{A}_{\mathrm{i}}(\mathrm{t}) \approx \mathrm{A}_{\mathrm{i}}(\mathrm{t}+\mathrm{T})$ and $\mathrm{B}_{\mathrm{i}}^{(\mathrm{N})}(\mathrm{t}) / \mathrm{N} \approx \mathrm{B}_{\mathrm{i}}^{(\mathrm{N}+1)}(\mathrm{t}+\mathrm{T}) /(\mathrm{N}+1)$. Consideration of MWD with $\mathrm{k}_{\mathrm{t}} \mathrm{WT} \leqslant 1$ regardless of the period of pseudostationary regime establishment gives a false idea of the pattern of polymer molecules concentrations distribution under the pseudostationary state. Only with the constraint $k_{t} W T \gg 1$, when pseudostationary state is virtually achieved following the second pulse, the period of its attainment need not be accounted for.

Initiating of polymerization by pulsed radiation allows to substantially govern the pattern of MWD formed in the process of polymerization and to gain fractions of polymer molecules with certain average weight by appropriate selection of beam parameters. Thus, for example, when conducting polymerization by pulse packets, it is possible to obtain distributions typical for the period of establishment of polymerization pseudo-or quasistationary regime.

And finally, results of calculations (see Figure 4 and Figure 5) directly indicate the range of repetition rate of initiating pulses in which the more contrast MWD spectrum may be obtained.

\section{Acknowledgement}

The authors are grateful to E. N. Sobol and N. G. Taganov for helpful discussions. 


\section{References}

1. A. N. Orayevsky, V. P. Pimenov, A. A. Stepanov and V. A. Sheglov, Kvantovaya Elektronika 4, 711(1974).

2. A. P. Alexandrov, V. N. Genkin, M. S. Kitai, I. M. Smirnova and V. V. Sokolov, Kvantovaya Elektronika 7, 547(1977).

3. C. Decker, ACS Symp. Ser., N 266, 207 (1984).

4. O. F. Olaj, I. Bitai and G. Gleixner, Macrom. Chem. 186, 2569 (1985).

5. H. Brackmann, M. Buback and H. P. Vogele, Macrom. Chem. 187, 1977(1986).

6. M. Buback, H. Hippler, J. Schwer and H. P. Vogele, Macrom. Chem., Rapid Commun. 7, 261 (1986).

7. C. Decker and K. Moussa, Macromolecules 22, 4455(1989).

8. C. Decker, SPIE 1279, 50 (1990).

9. C. Carre, C. Decker, J. P. Fouassier and D. J. Lougnot, Laser Chem. 10, 349(1990).

10. C. E. Hoyle, C. H. Chang and M. A. Trapp, Macromolecules 22, 3607 (1989).

11. A. V. Evseev and A. N. Nikitin, Isvestia AN (ser. fiz.) 57, N12, 132(1993).

12. H.S. Bagdasarian, Theory of radical polymerization (in Russian), M., Nauka, 1966.

13. S. I. Kuchanov, Methods of kinetic calculations in chemistry of polymers (in Russian), M., Khimia, 1978.

14. V. P. Gowariker, N. V. Viswanathan and J. Sreedhan, Polymer Science, New Delhi, India, 1986.

15. O. F. Olaj, I. Bitai and F. Hinkelmann, Macrom. Chem. 188, 1689(1987).

16. M. Buback, B. Huckestein and U. Leinhos, Macrom. Chem., Rapid Commun. 8, 473 (1987).

17. O. F. Olaj and I. Bitai, Macrom. Chem., Rapid Commun. 9, 275(1988).

18. M. Buback, B. Huckestein and B. Ludwig, Macrom. Chem., Rapid Commun. 13, 1 (1992).

19. K. W. McLaughlin, D. D. Latham, C. E. Hoyle and M. A. Trapp, J. Phys. Chem., 93, 3643(1989).

20. C. E. Hoyle, M. A. Trapp, C. H. Chang, D. D. Latham and K. W. McLaughlin, Macromolecules 22, 3866(1989). 